\title{
ESPRESSO on VLT: An Instrument for Exoplanet Research
}

\author{
Jonay I. González Hernández, Francesco Pepe, Paolo Molaro and Nuno Santos
}

\begin{abstract}
ESPRESSO (Echelle SPectrograph for Rocky Exoplanets and Stable Spectroscopic Observations) is a VLT ultra-stable high resolution spectrograph that will be installed in Paranal Observatory in Chile at the end of 2017 and offered to the community by 2018 . The spectrograph will be located at the Combined-Coudé Laboratory of the VLT and will be able to operate with one or (simultaneously) several of the four $8.2 \mathrm{~m}$ Unit Telescopes (UT) through four optical Coudé trains. Combining efficiency and extreme spectroscopic precision, ESPRESSO is expected to gaining about two magnitudes with respect to its predecessor HARPS. We aim at improving the instrumental radial-velocity precision to reach the $10 \mathrm{~cm} \mathrm{~s}^{-1}$ level, thus opening the possibility to explore new frontiers in the search for Earth-mass exoplanets in the habitable zone of quiet, nearby G to M-dwarfs. ESPRESSO will be certainly an important development step towards high-precision ultra-stable spectrographs on the next generation of giant telescopes such as the E-ELT.
\end{abstract}

Jonay I. González Hernández

Instituto de Astrofísica de Canarias, Vía Láctea S/N, E-38205 La Laguna, Tenerife, Spain

e-mail: jonay@iac.es

Universidad de La Laguna (ULL), Dpto. Astrofisica, 38206 La Laguna, Tenerife, Spain

Francesco Pepe

Observatoire de lUniversité de Genéve, Ch. des Maillettes 51, CH-1290 Versoix, Switzerland

e-mail: Francesco.Pepe@unige.ch

Paolo Molaro

INAF Osservatorio Astronomico di Trieste, Via Tiepolo 11, I-34143 Trieste, Italy

e-mail:molarodoats.inaf.it

Nuno Santos

Instituto de Astrofísica e Ciências do Espaço, Universidade do Porto, CAUP, Rua das Estrelas, 4150-762 Porto, Portugal

Departamento de Fsica e Astronomia, Faculdade de Ciências, Universidade do Porto, Rua do Campo Alegre, 4169-007 Porto, Portugal

e-mail: nuno.santoseastro.up.pt 


\section{Introduction}

During the last decades the exoplanet science has become one of the most exciting research fields in modern astrophysics. The doppler spectroscopy or radial velocity (RV) technique (based on the determination of the projected velocity of stars in the direction of the line of sight) was the earliest method delivering the first detection of a low-mass companions (e.g. HD114762 b - Latham et al. 1989). This technique provided in 1995 the first discovery of a Jupiter-mass exoplanet orbiting the solar-type star 51 Pegasi (Mayor and Queloz 1995). After the discovery of 51 Peg $\mathrm{b}$, direct imaging, microlensing and specially transit searches both from the ground and space (Lissauer et al. 2014), together with the RV technique (Mayor et al.2014), have produced an increasing number of detections of planets and planetary systems. The number of confirmed exoplanets discovered as of May 2017 is about 3,600, among which $\sim 2700$ exoplanets have been detected using the transit technique, mostly from the space missions CoROT (Barge et al. 2006) and Kepler (Borucki et al. 2009). About 700 exoplanets have been discovered using the doppler technique on high-resolution spectrographs. These detections from both transit and RV techniques have yielded a significant statistical contribution to our understanding of exoplanet population, such as the frequency of planets (Mayor et al. 2011, Howard et al. 2012), for different host spectral types and at different orbital distances including the habitable zone (i.e. in orbits where water is retained in liquid form on the planet surface).

Now we know there is wide variety of planetary systems with different planetary masses, sizes, orbital periods, eccentricities and different configurations of mass/size-distances to the host stars. Most of the known planets appear to be in planetary systems and a significant fraction in multiple planet systems. Examples of complex planetary systems as the Solar System are the 7-planet system orbiting the G dwarf HD10180, detected using the RV technique with six confirmed planets in the mass range $11-65 M_{\mathrm{E}}$ and one planet candidate with a mass as small as 1.5 $M_{\mathrm{E}}$ (Lovis et al. 2011), or the recent discovery using transit technique from the ground of also seven planets with similar size as that of the Earth orbiting the late $\mathrm{M}$ dwarf TRAPPIST-1 (Gillon et al.2017). Those discoveries have required intensive and long-term monitoring of stars and the continuous development of astronomical instrumentation (Pepe et al.2014a). This together with significant improvement in the observational strategies has made exoplanet science quickly evolve from the discovery of giant planets even more massive than Jupiter to rocky planets with similar mass as that of the Earth. This is demonstrated by the recent discoveries of Kepler $78 \mathrm{~b}$ (Pepe et al. 2013), a planet with similar density as that of the Earth, and Proxima Centauri b (Anglada-Escudé et al. 2016), a planet with similar minimum mass as that of the Earth in the habitable zone of the closest star to the Sun.

Coupling the transit with the RV technique allows us to derive the mass and radius, and therefore to compute bulk density of exoplanets, a first step towards characterization of exoplanets. This in combination with detailed theoretical modelling allows us to get some insights about the bulk composition of some exoplanets and how they compared with the Earth composition (see Mayor et al. 2014; Lis- 
sauer et al. 2014, and references therein). The next step is to charaterize the atmospheres of exoplanets, using e.g high-resolution spectroscopy via transmission with a technique applied to some transiting giant planets to detect $\mathrm{Na}$ and $\mathrm{CO}$ in their atmospheres (Charbonneau et al. 2002; Snellen et al. 2008, 2010; Wyttenbach et al. 2015), or via spectroscopic detection of reflected light of exoplanets (Charbonneau et al. 1999; Martins et al. 2015).

Most of the known transiting exoplanets (namely those discovered by the Kepler space telescope) orbit faint targets, which make it difficult to study their atmospheres with the current facilities. The recently found transiting super-Earth like planet in the habitable zone of the faint M dwarf LHS 1140 (Dittmann et al.2017) is an example of the need for larger telescopes equipped with high-resolution spectrograph to investigate the atmospheres of such interesting exoplanets. In particular, the difficulty raises when trying to find transiting Earth-like planets orbiting at the habitable zones of bright host stars. It appears quite necessary to design new techniques able to study the atmospheres of exoplanets by using the reflected light (Snellen et al. 2014), combining a high-contrast adaptive optics (AO) system with a highresolution spectrograph to overcome the tiny planet-to-star flux ratio in two stages. First, the AO system should spatially resolves the planet from the host star enhancing the planet-to-star contrast at the planet location and second, the light beam at the planet location passes through the high-resolution spectrographs. This technique has been recently applied to model the possible planetary atmospheric signal of Proxima Cen b by coupling the SPHERE high-contrast imager (Beuzit et al. 2008) and the ESPRESSO spectrograph at VLT (Lovis et al.2017). The new generation of 20-40m giant telescopes such as E-ELT equipped with sophisticated AO systems and with stable high-precision high-resolution spectrographs will possibly be able to study the atmospheres of rocky exoplanets in the habitable zone (Snellen et al. 2015).

The need of instrumentation for exoplanet science on the 8.2-m Very Large Telescopes (VLT) was highlighted in the ESO (European Southern Observatory)ESA(European Space Agency) working report on extrasolar planets. In October 2007 the ESO Science Advisory Committee recommended the development of new second-generation VLT instrumentation, and later endorsed by the ESO Council. Among those instruments, ESPRESSO, a high-resolution ultra-stable spectrograph for the VLT combined-Coudé focus, was proposed. The ESPRESSO (Echelle SPectrograph for Rocky Exoplanets and Stable Spectroscopic Observations) project started with the kick-off meeting in February 2011. The ESPRESSO consortium is composed of: Observatoire Astronomique de l'Université de Genéve (project head, Switzerland); Instituto de Astrofísica e Ciências do Espaço/Universidade de Porto and Universidade de Lisboa (Portugal); INAF-Osservatorio Astronomico di Brera (Italy); INAF-Osservatorio Astronomico di Trieste (Italy); Instituto de Astrofísica de Canarias (Spain); Physikalisches Institut der Universität Bern (Switzerland). ESO participates to the ESPRESSO project as Associated Partner. The ESPRESSO instrument is expected to be commissioned at the VLT in Paranal Observatory in the fall 2017 and possibly offered to the community by 2018 . 


\section{Exoplanet science with ESPRESSO}

The main scientific drivers of ESPRESSO are: (i) search for rocky planets; (ii) measure the variation of physical constants; and (iii) analyse the chemical composition of stars in nearby galaxies. We refer to Pepe et al. (2014b) for details on (ii) and (iii), as well as very challenging additional scientific topics that ESPRESSO will be able to address.

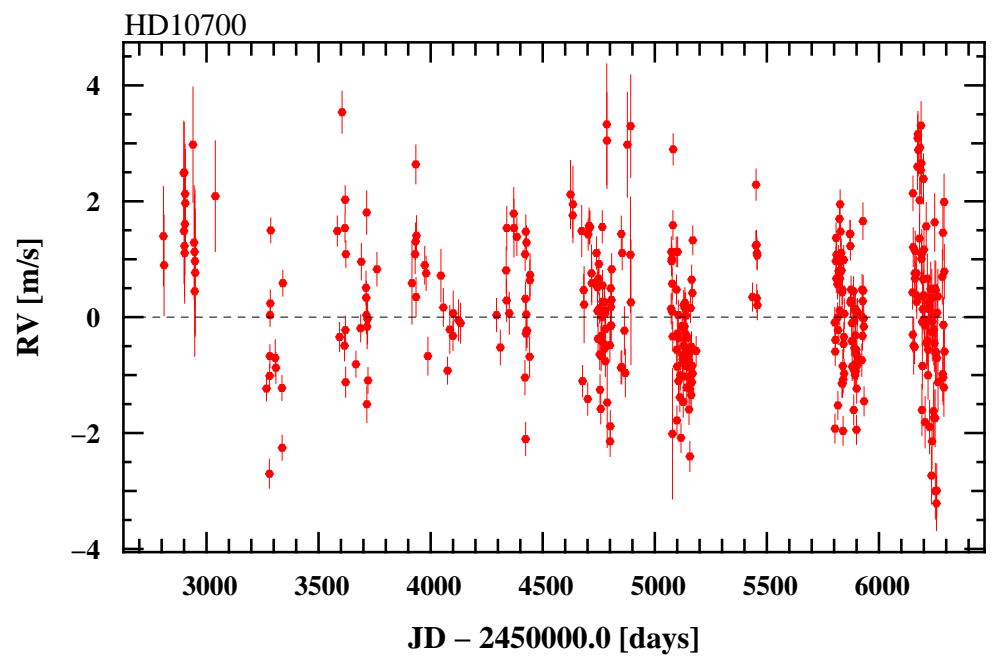

Fig. 1 Ten years HARPS RV data of the late G-type dwarf star $\tau$ Ceti. The overall dispersion is about $1 \mathrm{~m} \mathrm{~s}^{-1}$. Time-binning of the data would decrease the dispersion down to about $20 \mathrm{~cm} \mathrm{~s}^{-1}$. The absence of any ong-term trend in the data is remarkable, thus demonstrating the outstanding precision of the HARPS spectrograph. This figure has been taken from Pepe et al. (2014b).

One of the main scientific topics in the next decades is the search and charaterization of terrestrial planets in the habitable zone of their host stars, and one of the main drivers of the new generation of extremely large telescopes is the detection of their atmospheres (see e.g. Marconi et al. 2016). At the end of the 90's and the beginning of last decade, high-resolution spectroscopic monitoring of stars yielded many detections of Jupiter like planets. Only after 2003, the HARPS (Mayor et al. 2003) spectrograph installed at the 3.6-m ESO telescope in La Silla Observatory (Chile) opened a new window on the domain of Neptune and super-Earth like planets (see the Review by Pepe et al. in this HandBook of Exoplanets). This instrument (with a resolving power of $R \sim 115,000$ ) is contained inside a vacuum vessel and uses a simultaneous calibration reference that allows to correct for (small) instrumental drifts due to (also small) changes of temperature and pressure, providing an extreme long-term RV precision below the $1 \mathrm{~m} \mathrm{~s}^{-1}$ (see Fig. 1). Already in 2004 the discovery of a super-Earth with a minimum mass of about only $10 M_{\mathrm{E}}$ orbiting the 
G-type star $\mu$ Arae (Santos et al. 2004) demonstrated the impressive capabilities of the HARPS instrument. Since then, and thanks to its precision, HARPS has provided the discovery of most of the sub-Neptunian mass planets with masses down to a few Earth-masses inducing RV semi-amplitudes as low as $0.5 \mathrm{~m} \mathrm{~s}^{-1}$ (see e.g. Pepe et al. 2011).

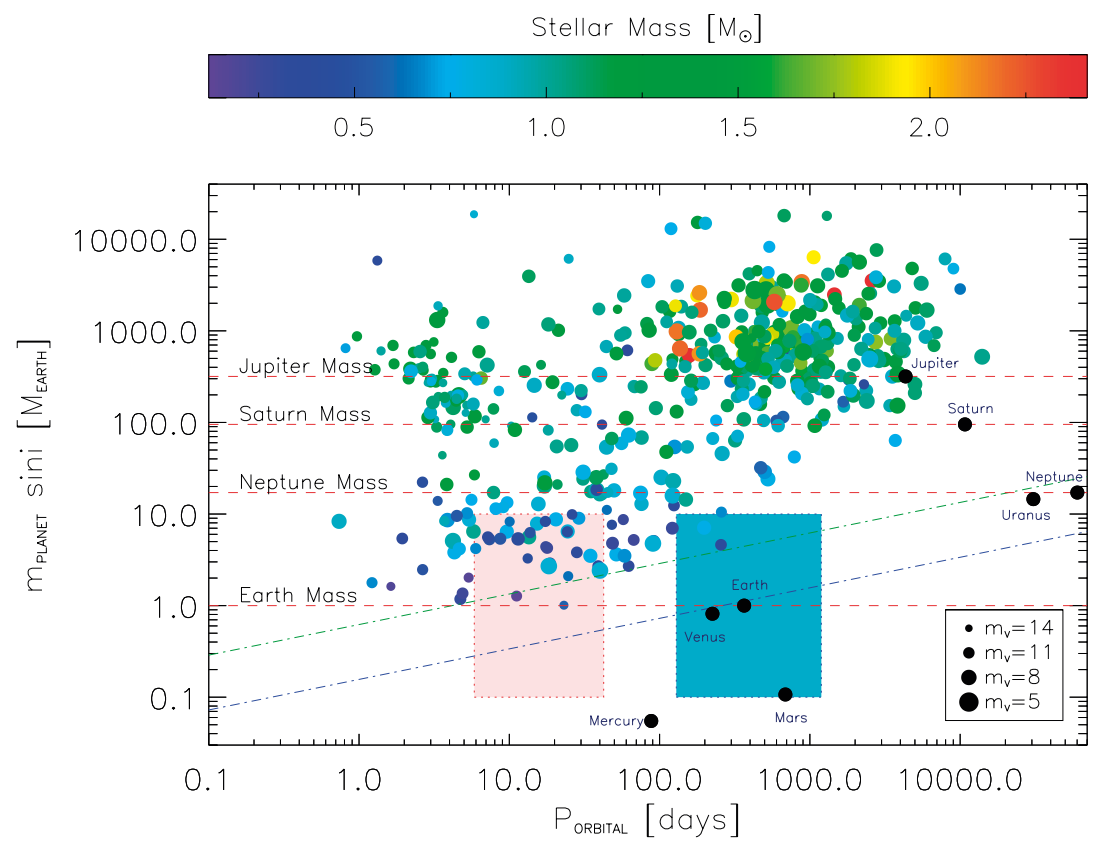

Fig. 2 Minimum mass - orbital period diagram for known planets orbiting solar-type stars. The color of the symbols is related to the mass of the host star given in solar mass $\left(\mathrm{M}_{\odot}\right)$ according to the color bar on top. Inclined dashed-dotted lines show the RV semiamplitude of planets orbiting a late $\mathrm{M}$ dwarf star with $0.25 \mathrm{M}_{\odot}$ (green line) and a $\mathrm{G}$ dwarf star with $1 \mathrm{M}_{\odot}$ star (blue line) assuming a RV semiamplitude of $1 \mathrm{~m} \mathrm{~s}^{-1}$ and $10 \mathrm{~cm} \mathrm{~s}^{-1}$, respectively, and null eccentricity. Planets of the solar system (filled black circles) are labeled. The "habitable zones" of $0.8-1.2 \mathrm{M}_{\odot}$ and $0.2-$ $0.3 \mathrm{M}_{\odot}$ stars are indicated with blue and pink rectangles, respectively. These are regions where rocky planets with a mass in the interval $0.1-10 M_{\mathrm{E}}$ would retain liquid water on their surface.

The ESPRESSO instrument will improve a step further, aiming at achieving a $\mathrm{RV}$ precision of $10 \mathrm{~cm} \mathrm{~s}^{-1}$ which is crucial in the path of detecting terrestrial planets in the habitable zone of host stars of different spectral types, in particular for $\mathrm{G}$, and $\mathrm{K}$ dwarfs. The Earth induces radial velocity variations in the Sun of $\sim 9 \mathrm{~cm} \mathrm{~s}^{-1}$, in comparison with RV signal of $12 \mathrm{~m} \mathrm{~s}^{-1}$ induced by Jupiter. However, an Earth-mass exoplanet orbiting a M5V star in the habitable zone would cause a gravitational pull equivalent to $\sim 1.3 \mathrm{~m} \mathrm{~s}^{-1}$ which, with the current instrument capabilities limited to $\sim 1 \mathrm{~m} \mathrm{~s}^{-1}$, can be detected (Bonfils et al. 2013a; Anglada-Escudé et al. 2016). Thus, the quest for low-mass planets in the habitable zone has favoured RV studies on $\mathrm{M}$ dwarf in the last decade with numerous low-mass planets detections (see e.g. 
Bonfils et al. 2013a|b; Anglada-Escudé et al. 2013, Affer et al. 2016; AstudilloDefru et al. 2017; Suárez Mascareño et al. 2017a|b). Today, several tens of planets with minimum masses below $10 M_{\mathrm{E}}$ have been discovered. Most of them have been detected orbiting cool dwarfs less massive than the Sun (see Fig. 2), using HARPS and HARPS-N (at 3.6-m TNG telescope located in the Observatorio del Roque de los Muchachos, La Palma, Spain; Cosentino et al. 2012) spectrographs. New dedicated instruments aiming for planet search around $\mathrm{M}$ dwarfs operating in the near infrared are already running such as CARMENES (at the 3.5-m telescope in the Observatorio de Calar Alto, Spain; Quirrenbach et al. 2016) or under construction such as NIRPS that will operate together with HARPS at the 3.6-m ESO telescope in La Silla (Chile), and SPIRou at the CHFT telescope in Mauna Kea (Hawaii, USA).

A larger telescope size provides a lower photon noise level on Doppler signal for the same exposure time. ESPRESSO at the 8.2-m VLT at the Observatorio de Paranal is expected to achieve the $10 \mathrm{~cm} \mathrm{~s}^{-1}$ Doppler precision and long-term stability. This will open the possibility to search for Earth-mass planets at different orbital distances, including the habitable zones of solar-type stars. A carefully selected sample of non-active, non-rotating, quiet $\mathrm{G}$ to $\mathrm{M}$ dwarf will allow to explore this new domain. In Fig. 2 we display the known planets at different orbital periods around stars with different apparent brightness. So far, most of the low-mass planets have been discovered around $\mathrm{M}$ dwarfs where the RV planetary signals are stronger. The larger telescope mirror of VLT and the RV precision provided by ESPRESSO will also allow to access a larger sample of fainter stars of different spectral types. However, stellar noise or jitter, which causes different radial velocity variations at different timescales and of different magnitude (see e.g. Saar et al. 1998; Santos et al. 2000: Queloz et al.2001; Boisse et al.|2011; Dumusque et al. 2011; Robertson et al. 2014, Suárez Mascareño et al. 2017c) still remains the main source of error and probably the strongest limitation towards the sub- $\mathrm{m} \mathrm{s}^{-1}$ precision. Therefore, continuous investigation and modelling on these stellar effects are required in the way towards finding rocky planets in the habitable zones of solar-type stars.

The discovery of a new and large population of Earth-mass exoplanets orbiting solar-type stars will expand our knowledge of planet formation, and will also deliver new candidates for follow-up observations using other techniques such as transit, astrometry, and Rossiter-McLaughlin (RM) effect. The detection with HARPS of the RM effect in occasion of the 2012 transit of Venus over the disk of the SUN provided a sort of preview of the kind of the physical information which we can hope to obtain by observing transits of exoplanets with a large telescope (Molaro et al. 2013b). ESPRESSO could also perform follow-up observations of ongoing and forthcoming transit surveys such as NGTS, MEarth from the ground or KeplerK2, TESS and Plato from the space. ESPRESSO will be possibly one of the rare instruments able to confirm long-period Earth-size transiting planets discovered by space missions like Plato, which hopefully will provide Earth-size candidates transiting bright targets in their habitable zones. The high resolution and stability of ESPRESSO will allow atmospheric characterization of exoplanets of different mass and size using the transmission and possibly reflection techniques (Charbonneau et al.|2002; Snellen et al. 2014; Martins et al.|2015, Lovis et al.|2017). 


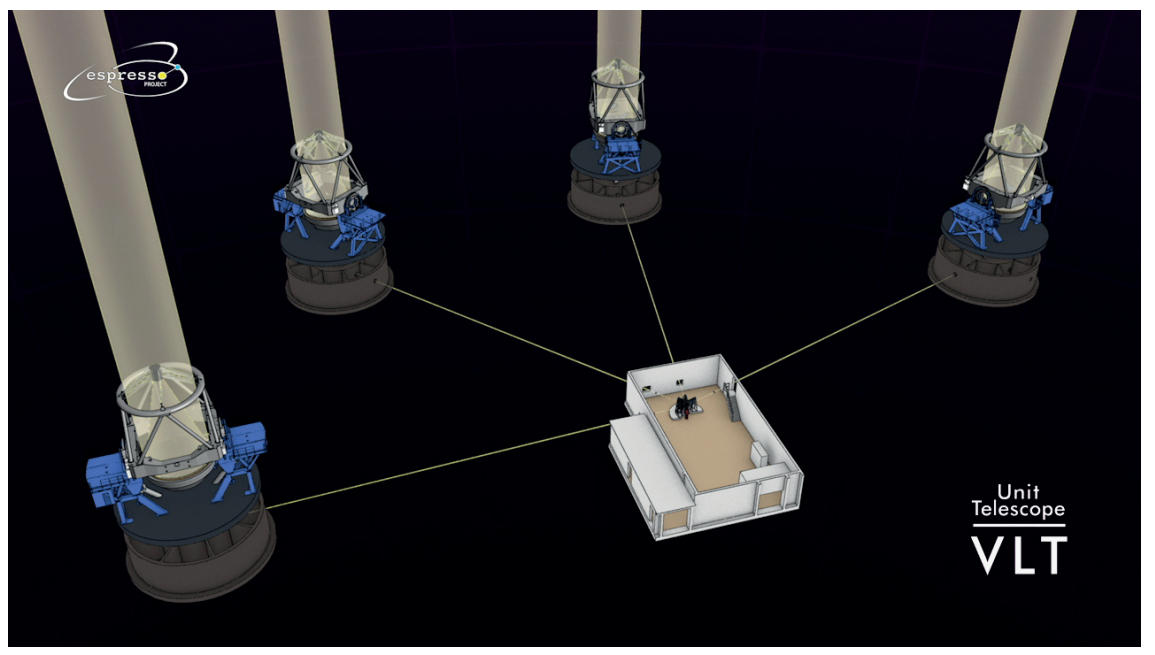

Fig. 3 Schematic view of the four $8.2 \mathrm{~m}$ Unit Telescopes of the VLT (multi-UT mode) feeding, through the Coudé train, the Front-End unit of the ESPRESSO spectrograph located in the Combined Coudé Laboratory.

\section{A ultra-stable high-resolution spectrograph for the VLT}

ESPRESSO is a fiber-fed, cross-dispersed, high-resolution échelle spectrograph located in the Combined Coudé Laboratory (CCL) at the incoherent focus, where a front-end unit can combine the light from up to four Unit Telescopes (UT) of the VLT. The so-called Coudé train optical system will fed the light of each UT to the spectrograph. The sky light and the target will enter the instrument simultaneously through two separate fibers, which form together the slit of the spectrograph. ESPRESSO, unlike any other ESO instrument, will be able to receive the light from any of the four 8.2-m UTs, and will be able to operate simultaneously with the light of either one UT or several UTs (see Fig. 3).

\section{Observing modes and performance}

The extreme precision of ESPRESSO will be achieved based on well-known concepts provided by the HARPS experience. The light of one or several UTs is fed through the front-end unit into optical fibers that scramble the light and provide excellent illumination stability to the spectrograph. In order to improve light scrambling, non-circular but octogonal or square fiber shapes are used (Chazelas et al. 2012).. The target fiber can be fed either with the light from the astronomical object or one of the calibration sources. The reference fiber will receive either sky light (faint source mode) or calibration light (bright source mode). In the latter case, 

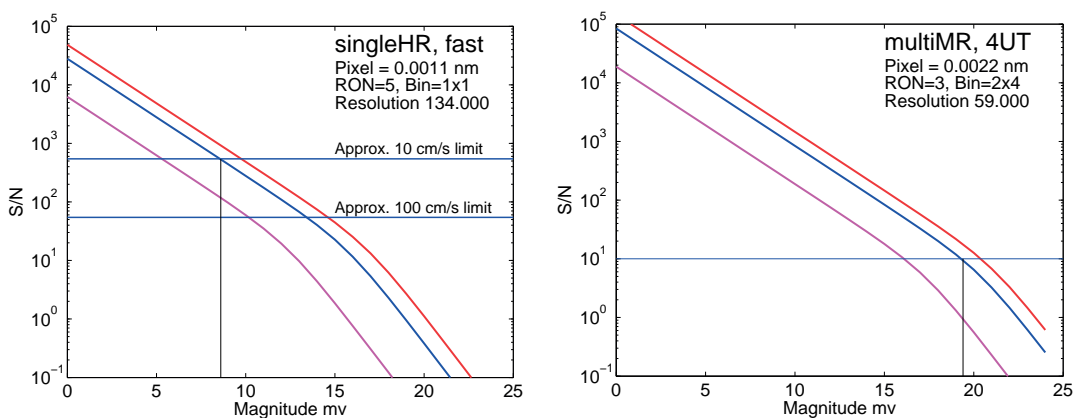

Fig. 4 Expected signal-to-noise ratio versus the stellar aparent visible magnitude for the singleHR mode (left panel) and the multiMR mode (right panel). Red, blue, and magenta curves indicate exposure times of $3600 \mathrm{~s}, 1200 \mathrm{~s}$ and $60 \mathrm{~s}$, respectively. This figure has been taken from Pepe et al. (2014b).

the simultaneous-reference technique succesfully applied in HARPS will enable to track instrumental drifts down to the $\mathrm{cm} \mathrm{s}^{-1}$ level. In this mode the measurement is photon-noise limited and detector read-out noise negligible. In the faint-source mode, instead, detector noise and sky background may become significant. In this case, the second fiber will allow to measure the sky background, whereas a slower read-out and high binning factor will reduce the detector noise. ESPRESSO will have three instrumental modes: singleHR, singleUHR and multiMR (see Table 1 ). Each mode will be available with two different detector read-out modes optimized for low and high-SNR measurements, respectively.

Table 1 Observing modes of ESPRESSO.

\begin{tabular}{lccc}
\hline Par./Mode & HR (1UT) & UHR (1UT) & MR(1-4UTs) \\
\hline Wave. range & $380-780 \mathrm{~nm}$ & $380-780 \mathrm{~nm}$ & $380-780 \mathrm{~nm}$ \\
Resol. Power & 134000 & $\approx 200000$ & 59000 \\
Aper. on Sky & $1.0 "$ & $0.5 ”$ & 4 ” $\times 1 "$ \\
Spec. Samp. & 4.5 pix & 2.5 pix & 11 pix \\
Spat. Samp. & $11 \times 2$ pix & $5 \times 2$ pix & $22 \times 2$ pix \\
Sim. Ref. & Yes (no sky) & Yes (no sky) & Yes (no sky) \\
Sky Sub. & Yes (no ref.) & Yes (no ref.) & Yes (no ref.) \\
Tot. Eff. & $11 \%$ & $5 \%$ & $11 \%$ \\
\hline
\end{tabular}

The observational efficiency of ESPRESSO is shown in Figs. 4. In the singleHR mode $(R \approx 134000)$, we estimate SNR $=10$ per extracted pixel in 20 minutes on a $V=16.3$ star, or a SNR $=540$ on a $V=8.6$ star. We have estimated that at this resolution and a SNR $=540$ will lead to $10 \mathrm{~cm} \mathrm{~s}^{-1} \mathrm{RV}$ precision for a non-rotating K5 star. For an F8 star, the same precision would be achieved for $V=8$. In the multiMR mode, at $R \approx 59000$, a SNR of $\approx 10$ is achieved on a $V=19.4$ star with an exposure of 20 minutes, a binning $2 \times 4$, and a slow read-out of the CCD. In the 
following sections we briefly describe the several subsystems of the ESPRESSO project (see Pepe et al. 2014b).

\section{The Coudé train}

The four VLT telescope are connected to the CCL through four tunnels with a length that goes from 48 to 69 meters (see Fig. 33. A trade-off analysis among the different solutions to bring the light from the telescope to the CCL favored a full optical solution that includes prisms, mirrors and lenses. The selected design uses 11 optical elements (see Fig. 5). The Coudé train takes the light beam with a prism at the Nasmyth-B platform and conduct it through the UT mechanical structure down to the Coudé room below each UT using a set of six prims and mirrors. The light is then routed from the UT Coudé room to the CCL, using two large lenses along the existing incoherent light ducts. The four Coudé trains relay a field of 17 arc$\mathrm{sec}$ around the acquired astronomical target to the CCL. The four beams from four UTs are combined in the CCL, where mode selection and beam conditioning is performed by the fore-optics of the Front-End subsystem. At the time of writting, all four Coud Trains have been installed and tested in Paranal. They have shown to provide seeing limited images to the front end that is installed at the CCL.

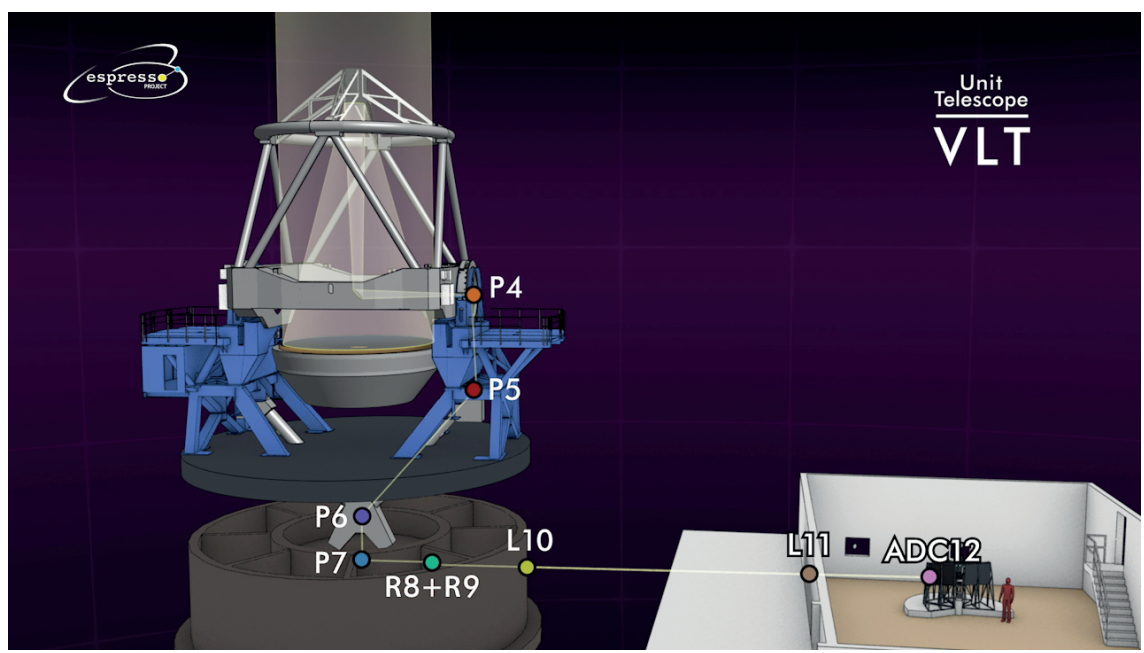

Fig. 5 Schematic view of the Coudé train of ESPRESSO with the optical path through the telescope to the Combined Coudé Laboratory. The optical elements are highlighted at different positions along the path from the Nasmyth-B platform to the CCL with P (prism), R (mirror) and L (lens). 


\section{The Front-End}

The Front-End conducts the light beam received at the CCL after correcting it for atmospheric dispersion with the ADC to the common focal plane where the spectrograph fiber feeds are located. A toggling mechanism handles the selection between the possible observational modes in a fully passive way. The beam conditioning is performed applying pupil and field stabilization (see Fig. 6). These are achieved via two independent control loops each consisting of a technical camera and a tiptilt stage. Another dedicated stage delivers a focusing function. The Front-End also handles the injection of the calibration light from the calibration unit into the fibers and then into the spectrograph. A laser frequency comb (LFC) system is foreseen as main calibration source. It produces a regular spectrum of lines equally spaced in frequency with an accuracy and stability linked to an atomic clock. The short-term Doppler shift repeatability of the LFC system has been tested in HARPS spectrograph and demonstrated to achieve the $\mathrm{cm} \mathrm{s}^{-1}$ level (Wilken et al. 2012, Probst et al. 2016). The required repeatability of the order of $\Delta \lambda / \lambda \approx 10^{-10}$ cannot be attained with currently used spectral sources such as thorium argon spectral lamps, iodine cells, etc, but can be obtained with a LFC that would provide a spectrum sufficiently wide, rich, stable and uniform for this purpose (Lo Curto et al. 2012; Molaro et al. 2013a). However, two ThAr lamps for both simultaneous reference and calibration will be also available as backup calibration sources, together with one simultaneous stabilized Fabry-Pérot unit, also as a backup solution.

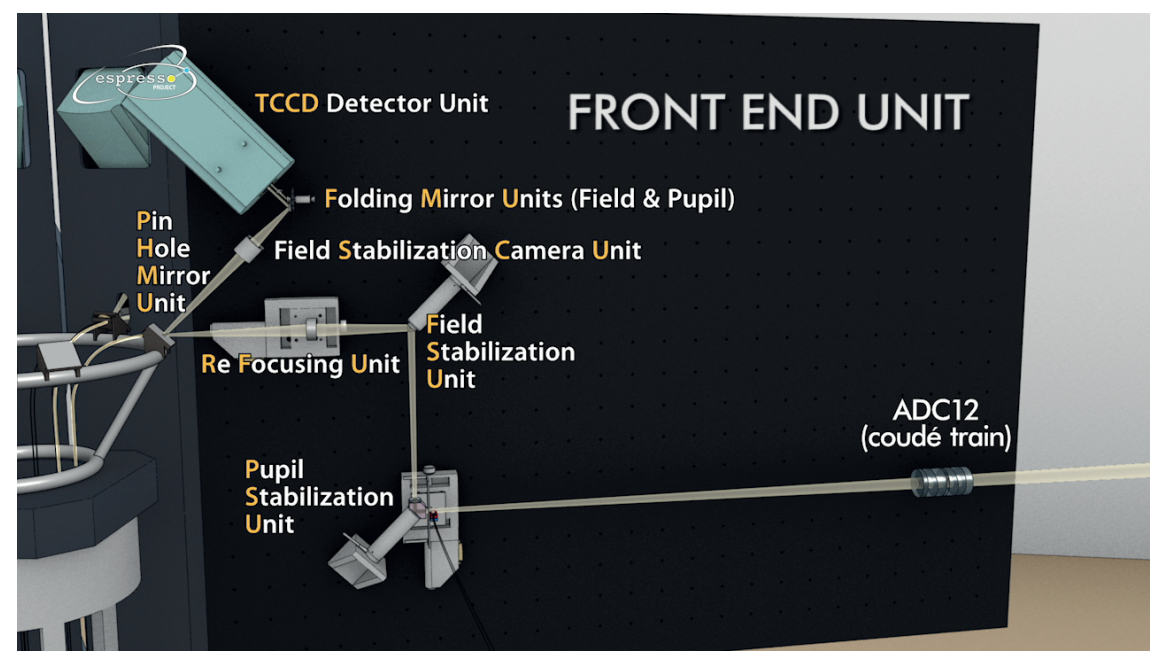

Fig. 6 Front-End unit and the arrival of one UT beam at the CCL. The same is replicated for the other UTs. 


\section{The Fiber-Link}

The Fiber-Link sybsystem transfers the light from the Front-End to the spectrograph and creates the a pseudo-slit in the output end inside the vacuum vessel. The 1-UT mode uses a bundle of two octogonal fibers each, one for the astronomical object and one for the sky or simultaneous reference. In the high-resolution mode (singleHR) mode, the fiber has a core of $140 \mu \mathrm{m}$, equivalent to 1 " on the sky; in the ultra-high resolution (singleUHR) mode the fiber core is $70 \mu \mathrm{m}$, covering a field of view of 0.5 ". The fiber entrances are organized in pickup heads that are moved to the focal plane of the Front End when the specific bundle of the specific mode is selected. In the 4-UT mode (multiMR) four object fibers and four sky/reference fibers are fed simultaneously by the four telescopes. The four object fibers and the four sky/reference fibers will finally feed two separate single square fibers of 280 $\mu \mathrm{m}$, for the object and for the sky/reference, respectively. In the 4-UT mode the spectrograph will also see a pseudo slit of four fiber square images twice as wide as the 1-UT fibers. One essential task performed by the Fiber-link subsystem is the light scrambling. The use of a double-scrambling optical system will ensure both scrambling of the near field and far field of the light beam. A high scrambling gain, which is crucial to obtain the required RV precision in the 1-UT mode is achieved by the use of octagonal fibers (Chazelas et al.2012).

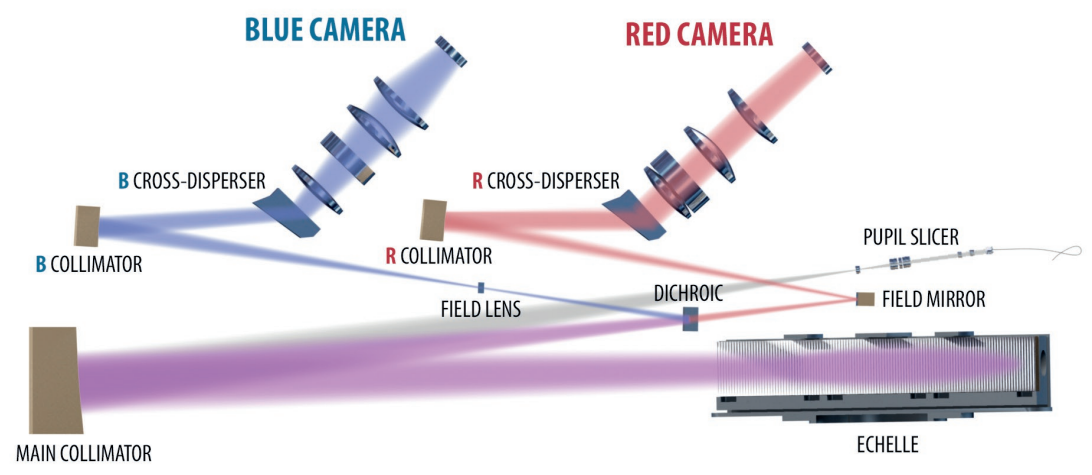

Fig. 7 Layout of the ESPRESSO spectrograph and its optical elements.

\section{Optical Design}

Designing a high efficiency and high resolution spectrograph is not an easy task due to the large mirrors of the VLT telescopes and the 1 arcsec aperture of the instrument. In order to minimize the size of the optics, and in particular, that of the 
main collimator and échelle grating, ESPRESSO implements an anamorphic optics, the APSU, which compresses the size of the pupil in the direction of the crossdispersion. The pupil is then sliced in two by a pupil slicer and the slices are overlapped on the échelle grating, leading to a doubled spectrum on the detector. This design reduces significantly the sizes of the optics and the échelle grating. Without this trick, the collimator beam size would have been $40 \mathrm{~cm}$ in diameter and the size of the échelle grating would have reached a size of $240 \times 40 \mathrm{~cm}$. The size of current échelle grating of ESPRESSO is only $120 \times 20 \mathrm{~cm}$ and this also allows the use of much smaller optics (collimators, cross dispersers, etc.). The échelle grating will be an R4 Echelle of $31.61 \mathrm{~mm}^{-1}$ and a blaze angle of $76^{\circ}$. This solution significantly reduces the overall costs. The drawback is that each spectral element will be covered by more detector pixels given the doubled image of the object fiber and its elongated shape on the CCD. In order to avoid to increase the detector noise, heavy binning will be done in the case of faint-object observations, especially in the 4-UT mode.

The main components of the optical design are (see Fig. 77):

- The Anamorphic Pupil Slicing Unit (APSU). At the spectrograph entrance the APSU shapes the beam in order to compress it in cross-dispersion and splits in two smaller beams, while superimposing them on the échelle grating to minimize its size. The rectangular white pupil is then re-imaged and compressed.

- Dichroic. Given the wide spectral range, a dichroic beam splitter separates the beam in a blue and a red arm, which in turn allows to optimize each arm for image quality and optical efficiency.

- Volume Phase Holographic Gratings (VPHGs). The cross-disperser enables to separate the dispersed spectrum in all its spectral orders. In addition, an anamorphism is re-introduced to make the pupil square and to compress the order height such that the inter-order space and the SNR per pixel are both maximized. Both functions are accomplished using Volume Phase Holographic Gratings (VPHGs) mounted on prisms.

- Fast Cameras. Two optimised camera lens systems image the full spectrum from $380 \mathrm{~nm}$ to $780 \mathrm{~nm}$ on two large $92 \times 92 \mathrm{~mm}$ CCDs with $10-\mu \mathrm{m}$ pixels.

A sketch of the optical layout is depicted in Fig. 7. The spectral format covered by the blue and the red chips as well as the shape of the pseudo slit are displayed in Fig. 8. In order to precisely compute the relative Earth motion to be able to properly correct the RV measurement, it is necessary to calculate the weighted mean time of exposure. Thus, the spectrograph is also equipped with an advanced exposure meter that measures the flux entering the spectrograph as a function of time. Its innovative design (based on a simple diffraction grating) allows a flux measurement and an RV correction at different spectral channels, in order to cope with possible chromatic effects that could occur during the scientific exposures. The use of various channels also provides a redundant and thus more reliable evaluation of the mean time of exposure. 


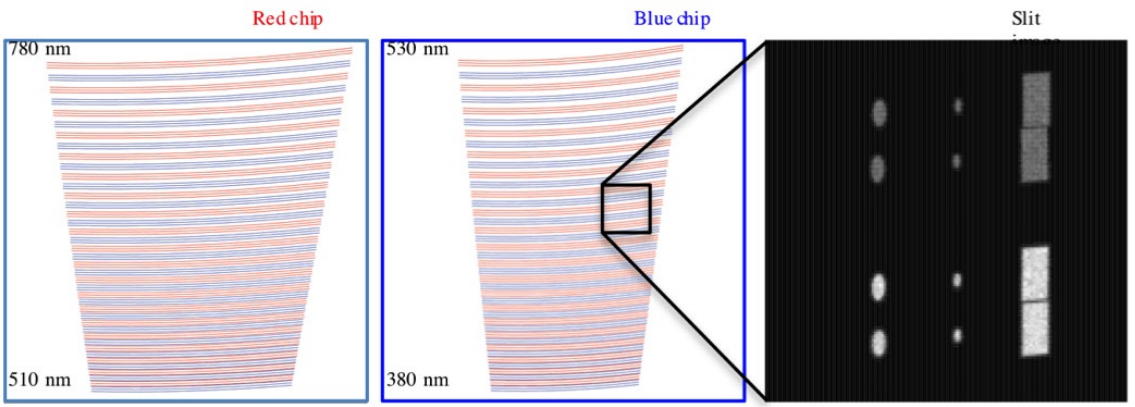

Fig. 8 Spectral format of the red (left panel) and blue (middle panel) spectra, and a zoom of the pseudo-slit (right panel), showing the image of the target (bottom) and sky (top) fibers. Each fiber is re-imaged into two slices. The three sets of fibers, corresponding (from left to right) to the standard resolution 1-UT mode, ultra-high resolution 1-UT mode and the mid-resolution 4-UT mode (shown simultaneously for comparison). This figure has been taken from Pepe et al. (2014b).

\section{The opto-mechanics}

ESPRESSO has been designed to be an ultra-stable spectrograph enabling RV precisions of the order of $10 \mathrm{~cm} \mathrm{~s}^{-1}$, i.e. one order of magnitude better than its predecessor HARPS. ESPRESSO is therefore built with a totally fixed configuration and with the highest thermo-mechanical stability. The spectrograph optics are mounted in an optical bench specifically designed to keep the optical system within the thermomechanical tolerances required for high-precision RV measurements. The bench is mounted in a vacuum vessel in which $10^{-5}$ mbar class vacuum is maintained during the entire duty cycle of the instrument. An overview of the opto-mechanics is shown in Fig. 9. The temperature at the level of the optical system is required to be stable at the $\mathrm{mK}$ level in order to avoid both short-term drift and long-term mechanical instabilities. Such an ambitious requirement is obtained by locating the spectrograph in a multi-shell active thermal enclosure system as shown in Fig. 10 Each shell will improve the temperature stability by a factor 10, thus getting from typically Kelvinlevel variations in the CCL down to $0.001 \mathrm{~K}$ stability inside the vacuum vessel and on the optical bench.

\section{Large-area CCDs}

The CCDs are another innovative solution in the ESPRESSO project. Large monolitic state-of-the-art CCDs have been chosen to use the optical field of ESPRESSO and to further improve the stability compared to the mosaic solution employed in HARPS. The sensitive area of the e $2 \mathrm{v}$ chip is $92 \times 92 \mathrm{~mm}$ covering $8.46 \times 10^{7}$ pixels of $10 \mu \mathrm{m}$ size. Fast read out of such a large chip is achieved by using its 16 output ports at high speed. Other requirements on CCDs are very demanding, e.g. in terms 


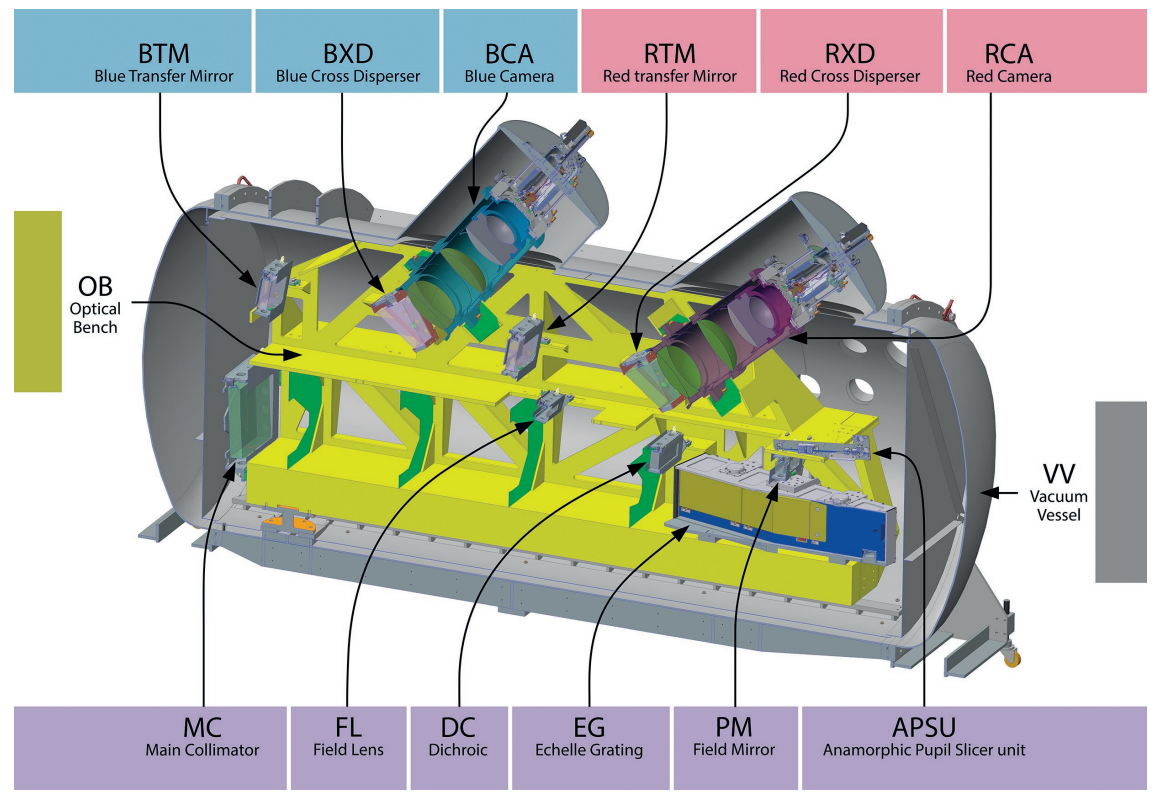

Fig. 9 Opto-mechanics of the ESPRESSO spectrograph.

of Charge Transfer Efficiency (CTE) and all the other parameters affecting the definition of the pixel position, immediately reflected into the radial-velocity precision and accuracy. The RV precision of $10 \mathrm{~cm} \mathrm{~s}^{-1} \mathrm{rms}$ requires measuring spectral line position changes of $2 \mathrm{~nm}$ (physical) in the CCD plane, equivalent to only 4 times the silicon lattice constant. For better stability and thermal-expansion matching the CCD package is made of silicon carbide. The package of the CCDs, the surrounding mechanics and precision temperature control inside the cryostat head and its cooling system, as well as the thermal stability and the homogeneous dissipation of the heat locally produced in the CCDs during operation are of critical importance. ESO has thus built a new superstable cryostat that has already demonstrated excellent short-term stability.

\section{Data flow system}

The ESPRESSO project has the final goal to provide the user with scientific data as complete and precise as possible in a short time (within minutes) after the end of an observation, to increase the overall efficiency and the ESPRESSO scientific output. For this purpose a software-cycle integrated view, from the observation preparation through instrument operations and control to the data reduction and analysis has been adopted. Coupled with a careful design this will ensure optimal compatibility and will facilitate the operations and maintenance within the existing ESO Paranal 


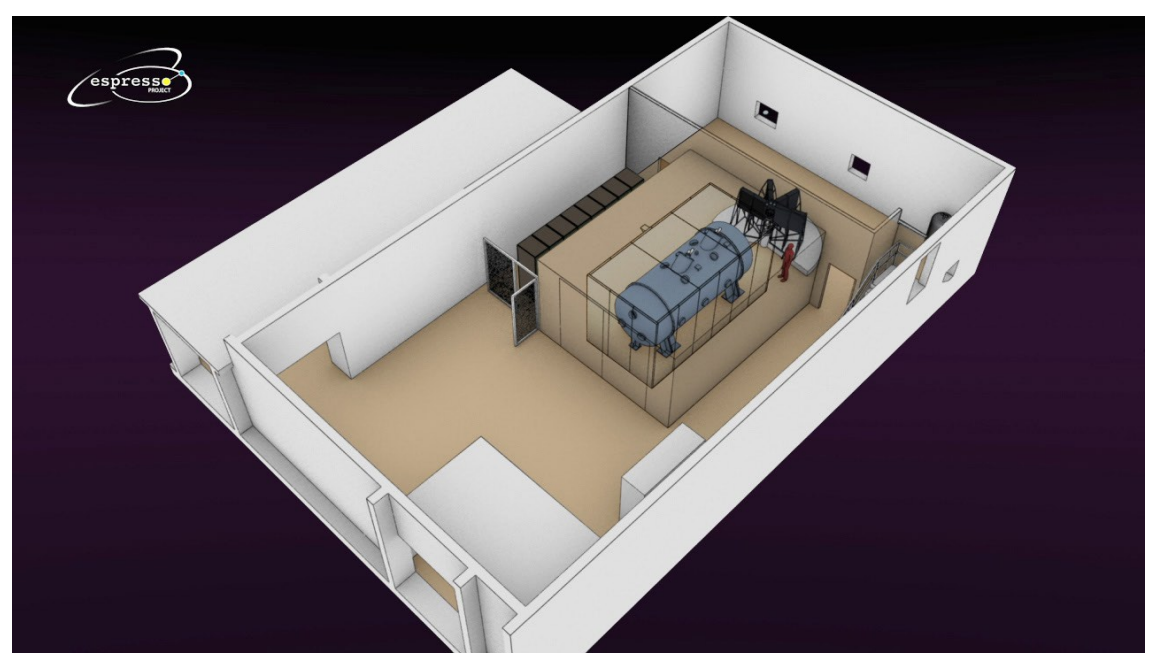

Fig. 10 Schematic view of the Combined Coudé Laboratory where ESPRESSO spectrogragh will be located inside the vacuum vessel, and several thermal enclosures in a multi-shell control system.

Data Flow environment both in service and visitor mode. ESPRESSO Data Flow System presents the following main subsystems:

- The ESPRESSO Observation Preparation Software (EOPS): a dedicated visitor tool (able to communicate directly with the VOT - Visitor Observing Tool) to help the observer to prepare and schedule ESPRESSO observations at the telescope according to the needs of planet-search surveys or other scientific programs. The tool will allow users to choose the targets best suited for a given night and to adjust the observation parameters in order to obtain the best possible quality of data.

- The Data Reduction Software (DRS): ESPRESSO will have a fully automatic data reduction pipeline with the specific aim of delivering to the user high-quality reduced data, science ready, already in a short time after an observation has been performed. The computation of the RV at a precision better than $10 \mathrm{~cm} / \mathrm{s}$ will be an integral part of the DRS. Coupled with the need to optimally remove the instrument signature, to take account the complex spectral and multi-HDU FITS format, the handling of the simultaneous reference technique and the multi-UT mode will make the DRS a truly challenging component of the DFS chain.

- The Data Analysis Software (DAS): dedicated data analysis software will allow to obtain the best scientific results from the observations directly at the telescope. A robust package of recipes tailored to ESPRESSO, taking full advantage of the existing ESO tools (based on CPL and fully compatible with Reflex), will address the most important science cases for ESPRESSO by analyzing (as automatically as possible) stars and quasar spectra (among others, tasks will be performed such as line Voigt-profile fitting, estimation of stellar atmospheric pa- 
rameters, normalization of stellar spectra and comparison with synthetic spectra, quasar continuum fitting, identification of absorption systems).

- Templates and control: compared to other standalone instruments, the main reason for the complexity of the ESPRESSO acquisition and observation templates will be the possible usage of any combination of UTs, besides the proper handling of the simultaneous reference technique. ESPRESSO will contribute to open the new path for the controlsystems of future ESO instrumentation.

\section{End-to-end operation}

ESPRESSO will combine an unprecedent RV and spectroscopic precision with the largest photon collecting area available today at the European Southern Observatory, and with an unique resolving power up to $R \sim 200,000$. In the singleHR mode, ESPRESSO can be fed with the light of an astromomical object coming from any of the four $8.2 \mathrm{~m}$ VLT telescopes, which significantly improves the scheduling flexibility for ESPRESSO programmes and surely will optimize the use of VLT time. The singleHR mode will operate at a resolution of 134,000 with a RV precision of $10 \mathrm{~cm} \mathrm{~s}^{-1}$, opening the possibility to explore a new population of rocky planets orbiting the habitable zones of solar-type stars. The scheduling flexibility is a fundamental advantage for survey programmes like RV searches for exoplanets or time-critical programmes like studies of transiting planets. The 1-UT mode also offer the possibility to enhance the resolving power up to 200,000 with a extremely stable wavelength accuracy which certainly will motivate new scientific projects e.g. high-accuracy stellar astrophysics projects. In addition, in the multiMR mode, ESPRESSO will be able to collect the light of up to four UTs to generate a highresolution spectrum. The effectively larger telescope aperture of about $16 \mathrm{~m}$ will provide access to faint astronomical targets at a resolution of 59,000. ESPRESSO shall not be considered as a stand-alone instrument but as a science-generating machine, certainly delivering full-quality scientific data in less than a minute after the end of an observation.

Acknowledgements The ESPRESSO project is supported by the Swiss National Science Foundation program FLARE, Italian Institute of Astrophysics (INAF), Instituto de Astrofísica de Canarias (IAC, Spain), Instituto de Astrofísica e Ciências do Espaço/Universidade de Porto and Universidade de Lisboa (Portugal), and the European Southern Observatory (ESO). J.I.G.H. acknowledges financial support from the Spanish Ministry of Economy and Competitiveness (MINECO) under the 2013 Ramón y Cajal programme MINECO RYC-2013-14875, and the Spanish ministry project MINECO AYA2014-56359-P. N.C.S. acknowledges the support by Fundação para a Ciência e a Tecnologia (FCT, Portugal) through the research grant through national funds and by FEDER through COMPETE2020 by grants UID/FIS/04434/2013\&POCI-01-0145-FEDER007672 and PTDC/FIS-AST/1526/2014\&POCI-01-0145-FEDER-016886, as well as through Investigador FCT contract nr. IF/00169/2012/CP0150/CT0002. The authors wish to acknowledge the exceptional work and enthusiasm delivered by all the members of the ESPRESSO team and warmly thank them for significantly contributing to the successful completion of the project. 


\section{References}

Affer L, Micela G, Damasso M et al. (2016) HADES RV program with HARPS-N at the TNG GJ 3998: An early M-dwarf hosting a system of super-Earths. A\&A593:A117

Anglada-Escudé G, Tuomi M, Gerlach E et al. (2013) A dynamically-packed planetary system around GJ 667C with three super-Earths in its habitable zone. A\&A556:A126

Anglada-Escudé G, Amado PJ, Barnes J et al. (2016) A terrestrial planet candidate in a temperate orbit around Proxima Centauri. Nature536:437-440

Astudillo-Defru N, Forveille T, Bonfils X et al. (2017) The HARPS search for southern extra-solar planets. XLI. A dozen planets around the M dwarfs GJ 3138, GJ 3323, GJ 273, GJ 628, and GJ 3293. A\&A602:A88

Barge P, Léger A, Ollivier M et al. (2006) Photometric Search for Transiting Planets. In: Fridlund M, Baglin A, Lochard J Conroy L (eds) The CoRoT Mission Pre-Launch Status - Stellar Seismology and Planet Finding, ESA Special Publication, vol 1306, p 83

Beuzit JL, Feldt M, Dohlen K et al. (2008) SPHERE: a 'Planet Finder' instrument for the VLT. In: Ground-based and Airborne Instrumentation for Astronomy II, Proc SPIE, vol 7014, p 701418, DOI 10.1117/12.790120

Boisse I, Bouchy F, Hébrard G et al. (2011) Disentangling between stellar activity and planetary signals. A\&A528:A4

Bonfils X, Delfosse X, Udry S et al. (2013a) The HARPS search for southern extra-solar planets. XXXI. The M-dwarf sample. A\&A549:A109

Bonfils X, Lo Curto G, Correia ACM et al. (2013b) The HARPS search for southern extra-solar planets. XXXIV. A planetary system around the nearby M dwarf ;ASTROBJ ${ }_{i}$ GJ $163_{i} / \mathrm{AS}$ TROBJ $_{i}$, with a super-Earth possibly in the habitable zone. A\&A556:A110

Borucki W, Koch D, Batalha N et al. (2009) KEPLER: Search for Earth-Size Planets in the Habitable Zone. In: Pont F, Sasselov D Holman MJ (eds) Transiting Planets, IAU Symposium, vol 253, pp 289-299, DOI 10.1017/S1743921308026513

Charbonneau D, Noyes RW, Korzennik SG et al. (1999) An Upper Limit on the Reflected Light from the Planet Orbiting the Star $\tau$ Bootis. ApJ522:L145-L148

Charbonneau D, Brown TM, Noyes RW Gilliland RL (2002) Detection of an Extrasolar Planet Atmosphere. ApJ568:377-384

Chazelas B, Pepe F Wildi F (2012) Optical fibers for precise radial velocities: an update. In: Modern Technologies in Space- and Ground-based Telescopes and Instrumentation II, Proc SPIE, vol 8450, p 845013, DOI 10.1117/12.926188

Cosentino R, Lovis C, Pepe F et al. (2012) Harps-N: the new planet hunter at TNG. In: Groundbased and Airborne Instrumentation for Astronomy IV, Proc SPIE, vol 8446, p 84461V, DOI $10.1117 / 12.925738$

Dittmann JA, Irwin JM, Charbonneau D et al. (2017) A temperate rocky super-Earth transiting a nearby cool star. Nature544:333-336

Dumusque X, Udry S, Lovis C, Santos NC Monteiro MJPFG (2011) Planetary detection limits taking into account stellar noise. I. Observational strategies to reduce stellar oscillation and granulation effects. A\&A525:A140

Gillon M, Triaud AHMJ, Demory BO et al. (2017) Seven temperate terrestrial planets around the nearby ultracool dwarf star TRAPPIST-1. Nature542:456-460

Howard AW, Marcy GW, Bryson ST et al. (2012) Planet Occurrence within 0.25 AU of Solar-type Stars from Kepler. ApJS201:15

Latham DW, Stefanik RP, Mazeh T, Mayor M Burki G (1989) The unseen companion of HD114762 - A probable brown dwarf. Nature339:38-40

Lissauer JJ, Dawson RI Tremaine S (2014) Advances in exoplanet science from Kepler. Nature513:336-344

Lo Curto G, Pasquini L, Manescau A et al. (2012) Astronomical Spectrograph Calibration at the Exo-Earth Detection Limit. The Messenger 149:2-6 
Lovis C, Ségransan D, Mayor M et al. (2011) The HARPS search for southern extra-solar planets. XXVIII. Up to seven planets orbiting HD 10180: probing the architecture of low-mass planetary systems. A\&A528:A112

Lovis C, Snellen I, Mouillet D et al. (2017) Atmospheric characterization of Proxima b by coupling the SPHERE high-contrast imager to the ESPRESSO spectrograph. A\&A599:A16

Marconi A, Di Marcantonio P, D'Odorico V et al. (2016) EELT-HIRES the high-resolution spectrograph for the E-ELT. In: Ground-based and Airborne Instrumentation for Astronomy VI, Proc SPIE, vol 9908, p 990823, DOI 10.1117/12.2231653

Martins JHC, Santos NC, Figueira P et al. (2015) Evidence for a spectroscopic direct detection of reflected light from $i$ ASTROBJ $_{i} 51$ Pegasi $b_{i} /$ ASTROBJ $_{i}$. A\&A576:A134

Mayor M Queloz D (1995) A Jupiter-mass companion to a solar-type star. Nature378:355-359

Mayor M, Pepe F, Queloz D et al. (2003) Setting New Standards with HARPS. The Messenger 114:20-24

Mayor M, Marmier M, Lovis C et al. (2011) The HARPS search for southern extra-solar planets XXXIV. Occurrence, mass distribution and orbital properties of super-Earths and Neptunemass planets. ArXiv e-prints

Mayor M, Lovis C Santos NC (2014) Doppler spectroscopy as a path to the detection of Earth-like planets. Nature 513:328-335

Molaro P, Esposito M, Monai S et al. (2013a) A frequency comb calibrated solar atlas. A\&A560:A61

Molaro P, Monaco L, Barbieri M Zaggia S (2013b) Detection of the Rossiter-McLaughlin effect in the 2012 June 6 Venus transit. MNRAS429:L79-L83

Pepe F, Lovis C, Ségransan D et al. (2011) The HARPS search for Earth-like planets in the habitable zone. I. Very low-mass planets around ;ASTROBJ ${ }_{i}$ HD 20794j/ASTROBJ ${ }_{i}$, ;AS-

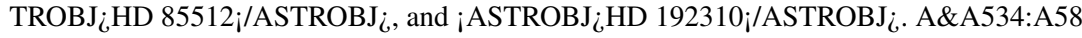

Pepe F, Cameron AC, Latham DW et al. (2013) An Earth-sized planet with an Earth-like density. Nature503:377-380

Pepe F, Ehrenreich D Meyer MR (2014a) Instrumentation for the detection and characterization of exoplanets. Nature513:358-366

Pepe F, Molaro P, Cristiani S et al. (2014b) ESPRESSO: The next European exoplanet hunter. Astronomische Nachrichten 335:8

Probst RA, Lo Curto G, Ávila G et al. (2016) Relative stability of two laser frequency combs for routine operation on HARPS and FOCES. In: Ground-based and Airborne Instrumentation for Astronomy VI, Proc SPIE, vol 9908, p 990864, DOI 10.1117/12.2231434

Queloz D, Henry GW, Sivan JP et al. (2001) No planet for HD 166435. A\&A379:279-287

Quirrenbach A, Amado PJ, Caballero JA et al. (2016) CARMENES: an overview six months after first light. In: Ground-based and Airborne Instrumentation for Astronomy VI, Proc SPIE, vol 9908, p 990812, DOI 10.1117/12.2231880

Robertson P, Mahadevan S, Endl M Roy A (2014) Stellar activity masquerading as planets in the habitable zone of the M dwarf Gliese 581. Science 345:440-444

Saar SH, Butler RP Marcy GW (1998) Magnetic Activity-related Radial Velocity Variations in Cool Stars: First Results from the Lick Extrasolar Planet Survey. ApJ498:L153-L157

Santos NC, Mayor M, Naef D et al. (2000) The CORALIE survey for Southern extra-solar planets. IV. Intrinsic stellar limitations to planet searches with radial-velocity techniques. A\&A361:265-272

Santos NC, Bouchy F, Mayor M et al. (2004) The HARPS survey for southern extra-solar planets. II. A 14 Earth-masses exoplanet around $\mu$ Arae. A\&A426:L19-L23

Snellen I, de Kok R, Birkby JL et al. (2015) Combining high-dispersion spectroscopy with high contrast imaging: Probing rocky planets around our nearest neighbors. A\&A576:A59

Snellen IAG, Albrecht S, de Mooij EJW Le Poole RS (2008) Ground-based detection of sodium in the transmission spectrum of exoplanet HD 209458b. A\&A487:357-362

Snellen IAG, de Kok RJ, de Mooij EJW Albrecht S (2010) The orbital motion, absolute mass and high-altitude winds of exoplanet HD209458b. Nature465:1049-1051 
Snellen IAG, Brandl BR, de Kok RJ et al. (2014) Fast spin of the young extrasolar planet $\beta$ Pictoris b. Nature 509:63-65

Suárez Mascareño A, González Hernández JI, Rebolo R et al. (2017a) A super-Earth orbiting the nearby M dwarf GJ 536. A\&A597:A108

Suárez Mascareño A, González Hernández JI, Rebolo R et al. (2017b) HADES RV Programme with HARPS-N at TNG. V. A super-Earth on the inner edge of the habitable zone of the nearby M dwarf GJ 625. A\&A605:A92

Suárez Mascareño A, Rebolo R, González Hernández JI Esposito M (2017c) Characterization of the radial velocity signal induced by rotation in late-type dwarfs. MNRAS468:4772-4781

Wilken T, Curto GL, Probst RA et al. (2012) A spectrograph for exoplanet observations calibrated at the centimetre-per-second level. Nature485:611-614

Wyttenbach A, Ehrenreich D, Lovis C, Udry S Pepe F (2015) Spectrally resolved detection of sodium in the atmosphere of HD 189733b with the HARPS spectrograph. A\&A577:A62 